\title{
Optimal Sampling of Nonstationary Spatiotemporal Fields Using a Mobile Sensor Network
}

\author{
Nitin Sydney*, Derek A. Paley** \\ * Department of Aerospace Engineering, University of Maryland, \\ College Park, MD, 20742 (email: nsydney@umd.edu) \\ ** Department of Aerospace Engineering, University of Maryland, \\ College Park, MD, 20742 (email: dpaley@umd.edu)
}

\begin{abstract}
This paper provides a decentralized control algorithm for multiple autonomous vehicles to sample environmental quantities on closed paths. We present a multi-vehicle control algorithm that samples a nonstationary spatiotemporal field using optimal interpolation to evaluate the sampling performance. The control algorithm leverages a coordinate transformation under which uniform sampling is optimal, because the unknown field is stationary in the new coordinates. The algorithm regulates the spacing between vehicles in order to limit the local maximum mapping error of the field reconstruction and preserves the steady-state vehicle sampling speed, which is a nondimensional measure of vehicle speed scaled by the spatial and temporal decorrelation lengths of the field at the vehicles location. The sampling performance is illustrated using a numerical simulation in a hypothetical environmental field.
\end{abstract}

Keywords: Decentralized control, nonstationary sampling, optimal control

\section{INTRODUCTION}

In environmental monitoring it is important to generate accurate spatiotemporal maps of scalar fields such as temperature (Inoue et al., 2005) or pollutant concentration (Torres et al., 2008). In physical oceanography, for example, it is often desired to sample along the boundary of a domain in order to determine the mass, heat, or salt budget (Rudnick and Davis, 1988). Although this task may be difficult to accomplish with a limited number of stationary sensors, using a mobile sensor network on a closed path has proved to be an effective solution (Leonard et al., 2010). Sampling performance is optimal (in the sense of minimizing mapping error) if measurements are concentrated in proportion to the variability of the unknown field.

Several approaches to sampling spatiotemporal fields exist in the literature. In distributed parameter estimation (Demetriou, 2010; Song et al., 2005; Ucinski and Demetriou, 2004), the mapping task is to determine the unknown parameters of a partial differential equation. With this approach, and other methods such as those based on optimal interpolation, a priori knowledge of the field is typical. For example, each vehicle may possess knowledge of the first and second-order statistics of the field in order to execute a routing strategy that minimizes mapping error (Davis et al., 2009; Sydney and Paley, 2011; Leonard et al., 2007). Recent works (Martinez et al., 2007; Laventall and Cortes, 2009; Zhang and Leonard, 2010) provide algorithms to increase mapping accuracy using mobile

\footnotetext{
* This material is based upon work supported by the National Science Foundation under Grant No. CMMI0928416 and the Office of Naval Research under Grant No. N00014-09-1-1058.
}

sensor platforms, though these algorithms are designed for stationary fields.

In contrast to previous works (Sydney and Paley, 2011; Paley, 2007), the focus of this paper is sampling a nonstationary spatiotemporal field, i.e., a spatiotemporal field in which the space-time statistics change when shifted in space or time. Many environmental quantities, such as pollutant concentration, have variable spatial structure and should be modeled as a nonstationary field. Some recent works on sampling nonstationary fields focus on coverage control (Cortez et al., 2004; Leonard and Olshevskyi, 2011), and use a heuristic measure of sampling need to measure sampling performance. For example, one algorithm (Leonard and Olshevskyi, 2011) samples a time-invariant field by routing vehicles to fixed locations. Other works (Sampson and Guttorp, 1992; Schmidt and O'Hagan, 2003) estimate the covariance structure of a nonstationary spatial field using fixed measurement locations. In one such work (Sampson and Guttorp, 1992), a coordinate transformation is used to ensure the covariance structure of the transformed field is stationary.

In this paper we provide an algorithm to sample nonstationary spatiotemporal fields using multiple mobile sensors on a closed path. We use intuition gained from the optimal mapping of a stationary field to develop the sampling method. We design a coordinate transformation that renders the field locally stationary in the transformed coordinates so that uniform coverage is optimal. We apply existing phase-control strategies (Paley and Peterson, 2009; Sydney and Paley, 2011) to equalize the vehicle spacing in the new coordinates. Since the mapping error is stationary in the transformed coordinates, we are able to determine 
the locations of local maximum error in the field. We show that this control strategy corresponds to preserving the steady-state sampling speed of the vehicles, which allows us to design sampling trajectories using the same methodology used for stationary fields. The sampling speed is a nondimensional measure of vehicle speed scaled by the spatial and temporal decorrelation lengths of the field at the vehicles location. In steady state, the vehicle speed varies to maintain its sampling speed. We illustrate the performance of the sampling strategy using a hypothetical environmental field.

The primary contribution of this paper is a decentralized multi-vehicle speed control to regulate inter-vehicle spacing so that a nonlinear coordinate transformation ensures the local mapping error is stationary. We also provide the location of the maximum error in a nonstationary field for a given set of trajectories generated in a stationary coordinate system. Although the multi-vehicle speed control leverages existing tools for stabilization of vehicle patterns on closed paths, its novelty lies in the use of a coordinate transformation that make these tools suitable for sampling in a nonstationary field. Indeed we expect that the results from this paper may be useful for other sampling designs, such as Voronoi-based methods (Graham and Cortes, 2009).

Section 2 summarizes relevant research on multi-vehicle coordination and provides background information on computing mapping error. Section 3 presents a control law that drives multiple vehicles to an equally spaced moving formation with constant sampling speed and derives the location of the maximum mapping error in the spatiotemporal field. Section 4 summarizes the paper.

\section{BACKGROUND}

The goal of this paper is to design a multi-vehicle control strategy to effectively sample a nonstationary field along a closed path. Section 2.1 describes an existing decentralized control originally designed for a system of coupled phase oscillators that regulates the inter-vehicle spacing in a moving formation. Section 2.2 describes how to optimally interpolate measurements taken along the vehicle trajectories to generate estimates of the spatiotemporal field and the corresponding mapping error. These techniques are used to design and evaluate the performance of the sampling method presented in Section 3.

\subsection{Splay Control for Coupled Phase Oscillators}

Modeling the positions of the vehicle fleet on a closed path as a set of coupled phase oscillators allows us to uniformly cover the sample space, which we elect to do in a set of coordinates other than the original space-time coordinates. This section reviews the formulation of a splay control (Sepulchre et al., 2007), which uniformly spaces a set of phase oscillators around the unit circle. Let phase variable $\theta_{k} \in S^{1}$ denote the non-dimensional position of vehicle $k$ on a closed path where $k=1, \ldots, N$. The dynamics of vehicle $k$ are

$$
\dot{\theta}_{k}=u_{k}\left(\theta_{1}, \ldots, \theta_{N}\right)=u_{k}(\theta),
$$

where we drop the subscript to indicate that the quantity is an $N \times 1$ matrix, e.g., $\theta=\left[\theta_{1} \cdots \theta_{N}\right]^{T}$.
We would like drive solutions of (1) to a splay formation (Sepulchre et al., 2007), which is a configuration of equally spaced phases. Choosing the control law (Sepulchre et al., 2007)

$$
u_{k}=-\frac{K_{m}}{N} \sum_{j=1}^{N} \sum_{m=1}^{\left\lfloor\frac{N}{2}\right\rfloor} \frac{1}{m} \sin \left(m\left(\theta_{k}-\theta_{j}\right)\right),
$$

where $K_{m}>0$ and $\lfloor x\rfloor$ is the largest integer less than $x$, stabilizes the vehicles to a splay formation (Sepulchre et al., 2007, Theorem 7). Adding a constant drift vector field, $s_{0} \in \mathbb{R}$, to the right-hand side of (1), representing motion around the path, does not change this result. Note that (2) is a gradient of the potential

$$
U^{\prime}(\theta) \triangleq \sum_{m=1}^{\left\lfloor\frac{N}{2}\right\rfloor} K_{m} U_{m}(\theta), \quad K_{m}>0
$$

where $\lfloor x\rfloor$ is the largest integer less than $x$,

$$
U_{m}(\theta) \triangleq \frac{N}{2}\left|p_{m \theta}\right|^{2}, \quad \text { and } \quad p_{m \theta} \triangleq \frac{1}{m N} \sum_{j=1}^{N} e^{i m \theta_{j}} .
$$

We use (2) to regulate the spacing between vehicles in a nonstationary field in Section 3.

\subsection{Evaluating Mapping Error using Optimal Interpolation}

We use measurements of a spatiotemporal field to generate an estimate of an unknown field and the corresponding error map as follows. Let $A\left(r_{k}\right)$ represent a continuous scalar field, where $r_{k} \triangleq\left[\begin{array}{ll}\theta_{k} & t_{k}\end{array}\right]^{T} \in S^{1} \times \mathbb{R}^{+}$describes the space-time location of a point on a closed path. The mapping error is determined using optimal interpolation (Eliassen and Sawuer, 1954; Gandin, 1963), which treats $A\left(r_{k}\right)$ as a discrete random field. Let $\tilde{r}_{d}=\left[\begin{array}{ll}\tilde{\theta}_{d} & \tilde{t}_{d}\end{array}\right]^{T}$ be the space-time location of measurement $d=1, \ldots, D$ and $\varepsilon_{d}$ be measurement noise. The measurement is $z_{d}=A\left(\tilde{r}_{d}\right)+\varepsilon_{d}$. Let $E[\cdot]$ denote the expected value of a random variable. We assume $E\left[\varepsilon_{m} \varepsilon_{l}\right]=\tilde{\sigma}_{0} \delta_{m l}$ for all pairs $m$ and $l$, where $\tilde{\sigma}_{0}$ is the standard deviation of the measurement noise and $\delta_{m l}$ is the Kronecker delta equal to one if $m=l$ and zero otherwise.

Let $\bar{A}\left(r_{i}\right)$ be the mean of the random field at location $r_{i}, i=1, \ldots, M$, and $C\left(r_{i}, r_{j}\right)$ be the covariance of points $r_{i}$ and $r_{j}$. The least squares estimate $\hat{A}\left(r_{i}\right)$ of $A\left(r_{i}\right)$ is (Liebelt, 1976)

$$
\hat{A}\left(r_{i}\right)=\bar{A}\left(r_{i}\right)+\sum_{d=1}^{D} C\left(r_{i}, \tilde{r}_{d}\right)\left(\sum_{l=1}^{D}\left(M^{-1}\right)_{d l} z_{d}\right),
$$

where $M^{-1}$ is the inverse of the measurement covariance matrix and

$$
M_{d l}=E\left[z_{d} z_{l}\right]=C\left(\tilde{r}_{d}, \tilde{r}_{l}\right)+\tilde{\sigma}_{0} \delta_{d l} .
$$

Let $C_{e}$ denote the matrix of error covariances after assimilating the set of measurements $\tilde{r}=\left[\begin{array}{lll}\tilde{r}_{1} & \cdots & \tilde{r}_{D}\end{array}\right]^{T}$. We have (Gandin, 1963; Bretherton et al., 1976)

$$
\begin{aligned}
C_{e}\left(r_{i}, r_{j} ; \tilde{r}\right)= & C\left(r_{i}, r_{j}\right)- \\
& \sum_{d=1}^{D} \sum_{l=1}^{D} C\left(r_{i}, \tilde{r}_{d}\right)\left(M^{-1}\right)_{d l} C\left(r_{j}, \tilde{r}_{l}\right) .
\end{aligned}
$$


The mapping error at $r_{i}$ is the diagonal element $C_{e}\left(r_{i}, r_{i} ; \tilde{r}\right)$ of the error covariance matrix.

To model nonstationary fields, we adopt a nonstationary covariance function of the form (Higdon et al., 1999)

$$
\begin{aligned}
C\left(r_{i}, r_{j}\right)= & \frac{\left|\Sigma\left(r_{i}\right)\right|^{1 / 4}\left|\Sigma\left(r_{j}\right)\right|^{1 / 4}}{\left|\frac{\Sigma\left(r_{i}\right)+\Sigma\left(r_{j}\right)}{2}\right|^{1 / 2}} \exp \left[-\frac{1}{2}\left(r_{i}-r_{j}\right)^{T} \times\right. \\
& \left.\times\left(\frac{\Sigma\left(r_{i}\right)+\Sigma\left(r_{j}\right)}{2}\right)^{-1}\left(r_{i}-r_{j}\right)\right],
\end{aligned}
$$

where $\Sigma\left(r_{k}\right) \in \mathbb{R}^{2 \times 2}$ is a positive definite matrix that is continuous in $r_{k}$. We further assume $\Sigma\left(r_{k}\right)$ has the form $\Sigma\left(r_{k}\right)=\operatorname{diag}\left\{\sigma^{2}\left(\theta_{k}\right), \tau^{2}\left(t_{k}\right)\right\}$, so that the decorrelation scales are decoupled in space and time. The diagonal entries of $\Sigma\left(r_{k}\right)$ are the spatial and temporal decorrelation scales of the field, respectively, which determine the elliptical footprint of a measurement. As $\sigma\left(\theta_{k}\right)$ (or $\tau\left(t_{k}\right)$ ) increases, the area of reduced mapping error around a measurement location $\tilde{r}_{k}$ increases. If $\sigma\left(\theta_{k}\right)$ and $\tau\left(t_{k}\right)$ are constant, (5) reduces to a stationary covariance function.

Figure 1(a) shows the along-track mapping error of two vehicles traveling around a circle of radius two in a stationary field, which creates a helical pattern in the spacetime volume. Figure 1(b) shows the error map on a twodimensional view of the space-time domain; vehicles traveling counter clockwise around the circle exit on the right of the space-time domain $\left(\theta_{k}=2 \pi\right)$ and reappear on the left $\left(\theta_{k}=0\right)$. Figure $1(\mathrm{c})$ shows how using a constant speed sampling strategy can produce areas of large sampling error in a nonstationary field. This motivates the need for sampling strategies designed for nonstationary fields that contain varying spatial and temporal scales.

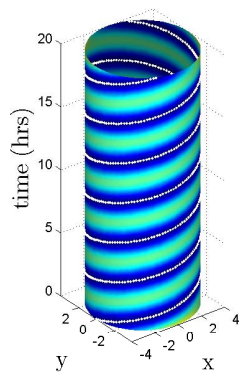

(a)

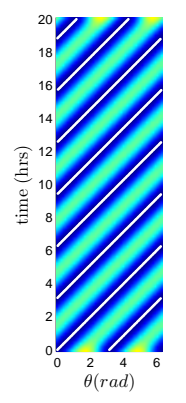

(b)

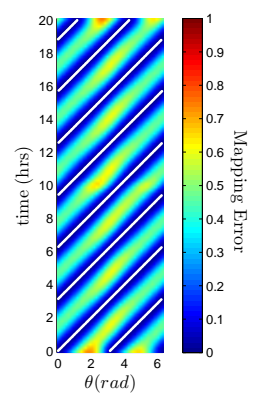

(c)
Fig. 1. Two vehicles traveling in a stationary field on a circular trajectory yield an error map represented (a) on the cylinder that encompasses the vehicle trajectories and (b) on a flattened representation of the space-time volume. (c) The error map for two constant-speed vehicles in a nonstationary field.

An important quantity in mapping error analysis is the non-dimensional vehicle speed $s_{k}$, called the sampling speed (Leonard et al., 2007), given for model (1) by

$$
s_{k}=\dot{\theta}_{k} \frac{\tau\left(t_{k}\right)}{\sigma\left(\theta_{k}\right)} .
$$

A vehicle's sensor swath (the portion of the domain covered by the set of sensor footprints generated along a sampling trajectory) is called spatially constrained if $s_{k}<1$ and temporally constrained if $s_{k}>1$ (Paley, 2007). Figures 2(a) and 2(b) compare the sensor swath of a spatially constrained vehicle to a temporally constrained one. The width of the sensor swath is determined by the spatial (resp. temporal) decorrelation scale for a spatially (resp. temporally) constrained vehicle. Figure 2(c) shows the variation of the sensor swath that occurs for a constantspeed vehicle in a nonstationary field, whose sampling speed is variable
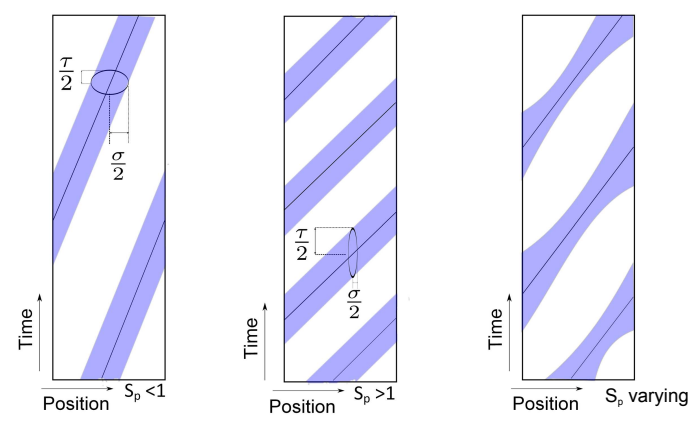

Fig. 2. Sensor swaths for (left) spatially constrained and (middle) temporally constrained vehicles in a stationary field; (right) sensor swath in a nonstationary field.

For a fleet of spatially (resp. temporally) constrained vehicles in a stationary field, a sampling strategy that covers the space-time domain is to separate the vehicles evenly in space (resp. time) (Sydney and Paley, 2011; Paley, 2007) as shown in Figure 1(b). The number of vehicles necessary to fully cover the domain is determined by the value of $s_{k}$ as follows (Paley, 2007). Let $\lceil x\rceil$ denote the smallest integer larger than $x$ and $T$ be the period of a single revolution around the path. If $s_{k}<1$, the number of vehicles need to cover the space is $N=\lceil 2 \pi / \sigma\rceil$, otherwise $N=\lceil T / \tau\rceil$ (Paley, 2007). These equations are simply the number of vehicles needed to tile the domain if the vehicle is spatially (resp. temporally) constrained. If the vehicle is spatially constrained. $N$ depends on the domain length and, if the vehicle is temporally constrained, $N$ depends on the domain time. The advantage of the algorithm in the following section is that it allows the application of this rubric to the problem of sampling a nonstationary field.

\section{ACHIEVING STATIONARY MAPPING ERROR USING SPLAY CONTROL}

In a stationary field, splay formations can be used to achieve optimal mapping performance (Sydney and Paley, 2011). In a nonstationary field, a splay formation may not be the optimal sampling strategy, as shown in Figure $1(\mathrm{c})$. In this section, we present a change of variables that renders a nonstationary field locally stationary. In the stationary coordinates, we optimize the mapping performance using a splay controller. We provide conditions for when the transformation exists and is invertible as well as conditions for the location of the maximum mapping error. We also show that a splay formation in the stationary coordinates corresponds to a time-varying formation in the nonstationary coordinates that preserves the steady-state vehicle sampling speed. 
Let $r_{k} \triangleq\left[\begin{array}{ll}\theta_{k} & t_{k}\end{array}\right]^{T}$ be the original coordinates and $y_{k} \triangleq$ $\left[\begin{array}{ll}\Psi_{k} & T_{k}\end{array}\right]^{T}$ be a set of transformed coordinates. We would like to find $y_{k}$ such that the field with covariance (5) becomes stationary, (after Sampson and Guttorp (1992)). Let $\bar{\Sigma}\left(r_{i}, r_{j}\right)=\left(\Sigma\left(r_{i}\right)+\Sigma\left(r_{j}\right)\right) / 2$ and $r_{i j}=r_{i}-r_{j}$. We seek $y_{k}$ that satisfies

$$
\begin{array}{r}
\frac{\left|\Sigma\left(r_{i}\right)\right|^{1 / 4}\left|\Sigma\left(r_{j}\right)\right|^{1 / 4}}{\sqrt{\left|\bar{\Sigma}\left(r_{i}, r_{j}\right)\right|}} \exp \left[-\frac{1}{2} r_{i j}^{T}\left(\bar{\Sigma}\left(r_{i}, r_{j}\right)\right)^{-1} r_{i j}\right] \\
=\exp \left[-\frac{1}{2}\left(y_{i}-y_{j}\right)^{T}\left(y_{i}-y_{j}\right)\right]
\end{array}
$$

for all pairs $i, j \in 1, \ldots, M$. Since we assume the decorrelation scales are decoupled in space and time, the transformations are decoupled too. Let $\Psi_{i j} \triangleq \Psi_{i}-\Psi_{j}$ and $T_{i j} \triangleq T_{i}-T_{j}$. Then, (7) yields

$$
\begin{aligned}
\Psi_{i j} & =\sqrt{\frac{2\left(\theta_{i}-\theta_{j}\right)^{2}}{\sigma^{2}\left(\theta_{i}\right)+\sigma^{2}\left(\theta_{j}\right)}+\ln \left(\frac{\sigma^{2}\left(\theta_{i}\right)+\sigma^{2}\left(\theta_{j}\right)}{2 \sqrt{\sigma^{2}\left(\theta_{i}\right) \sigma^{2}\left(\theta_{j}\right)}}\right)} \\
T_{i j} & =\sqrt{\frac{2\left(t_{i}-t_{j}\right)^{2}}{\tau^{2}\left(t_{i}\right)+\tau^{2}\left(t_{j}\right)}+\ln \left(\frac{\tau^{2}\left(t_{i}\right)+\tau^{2}\left(t_{j}\right)}{2 \sqrt{\tau^{2}\left(t_{i}\right) \tau^{2}\left(t_{j}\right)}}\right)} .
\end{aligned}
$$

Determining how to transform the domain such that the (8) and (9) hold for all $i$ and $j$ is a numerical analysis problem beyond the scope of this paper. Consider instead the special case where $j=i+1$, i.e., $\Psi_{j}=\Psi_{i}+\Delta \Psi$ and $\theta_{j}=\theta_{i}+\Delta \theta$. This corresponds to ensuring the domain is locally stationary about the $i$ th location. By locally stationary, we mean that only the covariance between adjacent locations are rendered stationary.

Lemma 1. A coordinate transformation that ensures the domain is locally stationary is

$$
\begin{aligned}
\Psi_{k} & =\int_{0}^{\theta_{k}} \frac{1}{\sigma\left(\theta^{\prime}\right)} d \theta^{\prime} \\
T_{k} & =\int_{0}^{t_{k}} \frac{1}{\tau\left(t^{\prime}\right)} d t^{\prime} .
\end{aligned}
$$

Furthermore, the transformations exist and are invertible if $\sigma\left(\theta_{k}\right)$ and $\tau\left(t_{k}\right)$ are positive, bounded, and continuous.

Proof. If $j=i+1$, i.e., $\Psi_{j}=\Psi_{i}+\Delta \Psi$ and $\theta_{j}=\theta_{i}+\Delta \theta$, then (8) reduces to

$$
\begin{aligned}
\Delta \Psi= & \left(\frac{2(\Delta \theta)^{2}}{\sigma^{2}\left(\theta_{i}\right)+\sigma^{2}\left(\theta_{i}+\Delta \theta\right)}+\right. \\
& \left.\ln \left(\frac{\sigma^{2}\left(\theta_{i}\right)+\sigma^{2}\left(\theta_{i}+\Delta \theta\right)}{2 \sqrt{\sigma^{2}\left(\theta_{i}\right) \sigma^{2}\left(\theta_{i}+\Delta \theta\right)}}\right)\right)^{1 / 2}
\end{aligned}
$$

We divide both sides by $\Delta \theta$ and take the limit as $\Delta \theta, \Delta \Psi \rightarrow 0$ to obtain

$$
\frac{\partial \Psi_{k}}{\partial \theta_{k}}=\frac{1}{\sigma\left(\theta_{k}\right)} .
$$

Integrating both sides yields the desired result. The derivation of the temporal transformation is similar.
If $\sigma_{k}$ and $\tau_{k}$ are bounded and continuous, then the integrals in both transformations exist. If the decorrelation scales are both positive, then $\Psi_{k}$ and $T_{k}$ are monotonically increasing functions of $\theta_{k}$ and $t_{k}$ and are hence invertible.

In the $\left(\Psi_{k}, T_{k}\right)$ coordinates, the mapping error is locally stationary, thus applying splay control in these coordinates optimizes sampling performance. In addition, the control is decentralized, so there is no need for a central unit to generate and transmit control commands. The following theorem provides a speed controller that prescribes uniform coverage of the field in the stationary coordinates.

Theorem 2. Let

$$
\phi_{k}=\frac{2 \pi}{\Psi_{k}(2 \pi)} \Psi_{k}\left(\theta_{k}\right)
$$

be a phase variable that is $2 \pi$ periodic. A speed control that drives (1) to a splay formation in the stationary coordinates and a time-varying formation with constant sampling speed $s_{0}$ in the original coordinates is

$$
u_{k}=\frac{\sigma\left(\theta_{k}\right)}{\tau\left(t_{k}\right)}\left(s_{0}-\frac{\partial U^{\prime}}{\partial \phi_{k}}\right),
$$

where $U^{\prime}(\phi)$ is given by (3) with $\theta$ replaced by $\phi$.

Proof. The dynamics of the $k$ th vehicle are

$$
\frac{d \Psi_{k}}{d T_{k}}=\nu_{k}\left(\Psi_{1}, \Psi_{2}, \ldots, \Psi_{N} ; T_{k}\right)
$$

To drive the vehicles to a splay formation, we choose

$$
\nu_{k}=s_{0}-\frac{\partial U^{\prime}}{\partial \phi_{k}},
$$

Using equations (10) and (11) we have

$$
u_{k}=\dot{\theta}_{k}=\frac{d \theta_{k}}{d \Psi_{k}} \frac{d \Psi_{k}}{d T_{k}} \frac{d T_{k}}{d t_{k}}=\frac{\sigma\left(\theta_{k}\right)}{\tau\left(t_{k}\right)}\left(s_{0}-\frac{\partial U^{\prime}}{\partial \phi_{k}}\right),
$$

which is the desired result.

In steady state, $\frac{\partial U^{\prime}}{\partial \phi_{k}}=0$ which implies

$$
\dot{\theta}_{k}=\frac{\sigma\left(\theta_{k}\right)}{\tau\left(t_{k}\right)} s_{0} .
$$

Solving for $s_{0}$ we see

$$
s_{0}=\frac{\tau\left(t_{k}\right)}{\sigma\left(\theta_{k}\right)} \dot{\theta}_{k}
$$

which is the sampling speed given by (6). Thus, the control (14) preserves the vehicles steady-state sampling speed $s_{0}$.

Once a splay formation is reached, the vehicle speed changes such that the sampling speed is constant. Theorem 2 allows us to design sampling trajectories according to the rubric given in Section 2.2, which determines the number of vehicles needed to fully cover the sampling domain. This sampling strategy guarantees coverage of a stationary field, it does not guarantee full coverage of a nonstationary one because the coordinate transformation is only valid locally as mentioned before Lemma 1 . So, although the full domain will not be covered, the constant samplingspeed strategy performs better in areas of high variability than a constant-speed strategy. 
In a stationary field, mapping error is reduced by increasing the sampling speed of the vehicle. The maximum attainable sampling speed is dictated by the maximum speed of the vehicle. Hence, we choose $s_{0}$ using (6) to be as large as possible without exceeding the maximum speed of the platform. In the transformed coordinates the mapping error is stationary, which implies the location of the maximum mapping error is can be determined. The following Lemma provides a necessary condition for the locations of maximum mapping error.

Lemma 3. Let $F: \theta_{i}\left(t_{i}\right) \rightarrow \Psi_{i}\left(T_{i}\right)$ be the mapping from the stationary coordinates to the nonstationary coordinates and $\tilde{\Psi}=\left[\tilde{\Psi}_{1}\left(T_{i}\right), \ldots, \tilde{\Psi}_{N}\left(T_{i}\right)\right]^{T}$ be the locations of the the $N$ vehicles at some time $T_{k}$ in the stationary coordinates. A necessary condition for the location of maximum mapping error at a specific time, $\theta_{i, \max }\left(t_{i}\right)$ is

$$
\sum_{k=1}^{N} \sum_{j=1}^{N} \frac{\partial C\left(\Psi_{i, \max }, \tilde{\Psi}_{j}\right)}{\partial \Psi_{i}}\left(M^{-1}\right)_{k j} C\left(\Psi_{i, \max }, \tilde{\Psi}_{k}\right)=0
$$

and

$$
\begin{aligned}
& \sum_{k=1}^{N} \sum_{j=1}^{N}\left[\frac{\partial^{2} C\left(\Psi_{i}, \tilde{\Psi}_{j}\right)}{\partial \Psi_{i}^{2}}\left(M^{-1}\right)_{k j} C\left(\Psi_{i, \max }, \tilde{\Psi}_{k}\right)+\right. \\
& \left.\frac{\partial C\left(\Psi_{i, \max }, \tilde{\Psi}_{j}\right)}{\partial \Psi_{i, \max }}\left(M^{-1}\right)_{k j} \frac{\partial C\left(\Psi_{i, \max }, \tilde{\Psi}_{k}\right)}{\partial \Psi_{i, \max }}\right]<0
\end{aligned}
$$

where $\Psi_{i, \max }=F\left(\theta_{i, \max }\right)$. The locations of local maximum error are values of $\theta_{i, \max }$ that satisfy (19) and (20).

The first condition comes from taking the first derivative of (4) and setting it equal to zero to locate the extreme points. The locations are guaranteed to be a minimum if the second derivative of (4) is less than zero at the extreme point, which is the second condition.

As an example of the overall sampling strategy, consider the following decorrelation-scale functions for $\sigma_{k}$ and $\tau_{k}$ :

$$
\begin{aligned}
\sigma_{k} & =\sigma_{0}-\mu_{1} e^{\mu_{2}\left(\cos \left(\theta_{k}-\theta_{0}\right)-1\right)}, \\
\tau_{k} & =\tau_{0}-\phi_{1} e^{-\phi_{2}\left(t_{k}-t_{0}\right)^{2}}
\end{aligned}
$$

where $\mu_{1}, \mu_{2}$, and $\theta_{0}$ are parameters that determine the size, shape, and location of a dip in the spatial decorrelation scale from the nominal value of $\sigma_{0}$; and $\alpha_{1}$, $\alpha_{2}$, and $t_{0}$ are parameters that determine the size, shape, and location of a dip in the temporal decorrelation scale from the nominal value of $\tau_{0}$. Figure 3 shows the temporal decorrelation scale as a function of $t_{k}$ and the transformed coordinate $T_{k}$, which is warped near $t_{0}$.

Figure 4(a) shows the decorrelation scales of a nonstationary field represented using ellipses in the space-time plane. Figure 4(b) shows how a uniform grid in the transformed coordinates looks under the transformation (10)-(11). In the original coordinates, space and time contract around a nonstationarity. This means a vehicle traveling at constant speed in the transformed coordinates will slow down near a nonstationarity to maintain its sampling speed, which places more measurements near the nonstationarity.

Using the parameters in Table 1, we conducted a simulation of two vehicles with arbitrary initial conditions in
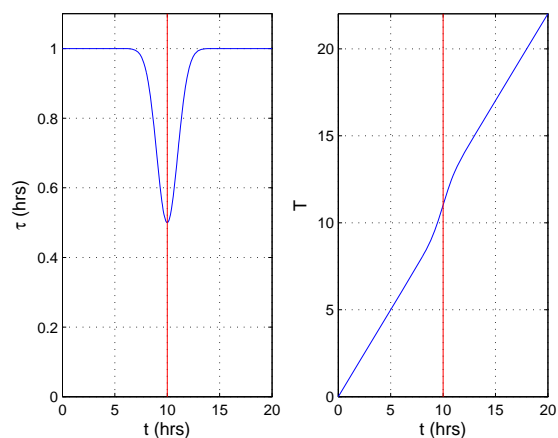

Fig. 3. Example of a nonstationary temporal decorrelation scale with nonstationarity centered at $t_{0}=10$. The shaping parameters are $\tau_{0}=1, \alpha_{1}=0.5$ and $\alpha_{2}=0.5$.

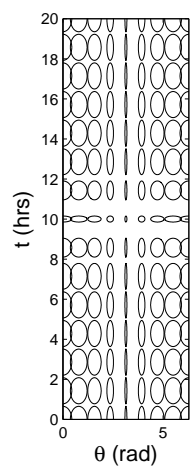

(a)

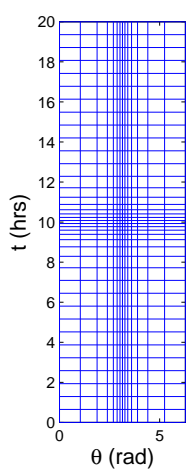

(b)
Fig. 4. (a) Ellipses showing the size of the decorrelation scales in a nonstationary field. (b) Transformation of a uniform grid under equations (10)-(11).

Table 1.

\begin{tabular}{|l|l|l|l|}
\hline \multicolumn{4}{|c|}{ Parameter Values } \\
\hline$\tau_{0}$ & 1.5 hours & $\sigma_{0}$ & $1.5 \mathrm{rad}$ \\
$\phi_{1}$ & 0.8 hours & $\mu_{1}$ & $0.7 \mathrm{rad}$ \\
$\phi_{2}$ & 0.5 hours & $\mu_{2}$ & 1.0 \\
$t_{0}$ & 10 hours & $\theta_{0}$ & $\pi \mathrm{rad}$ \\
$s_{0}$ & $0.6 \mathrm{rad} /$ hour & & \\
\hline
\end{tabular}

a hypothetical unknown field. Figure 5(a) shows the trajectories in the transformed coordinates generated using (1) with $u_{k}$ given by (14) and sampling speed $s_{0}=0.6$. Figure 5(b) shows the sampling trajectories in the original coordinates, which warp near the nonstationarity in order to maintain $s_{k}=s_{0}$. Figure 5 (c) shows the corresponding mapping error. The black x's indicate the locations of the maximum mapping error found using Lemma 3. The average mapping error using this control strategy is 0.20 whereas the average mapping error for the same number of vehicles traveling at constant speed is 0.24 (we used a constant speed equal to the average speed produced by the sampling-speed strategy.) Driving the vehicles to a constant sampling speed yields less mapping error.

\section{CONCLUSION}

This paper provides a multi-vehicle control algorithm for sampling a nonstationary spatiotemporal field on a closed path. The control strategy uses a coordinate transformation that makes the unknown field locally stationary. The 
(a)

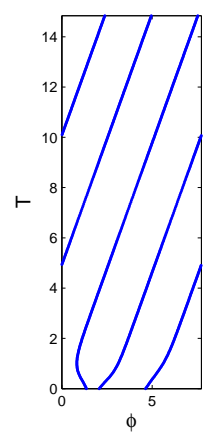

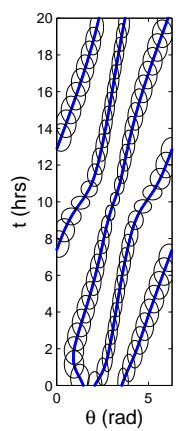

(b)

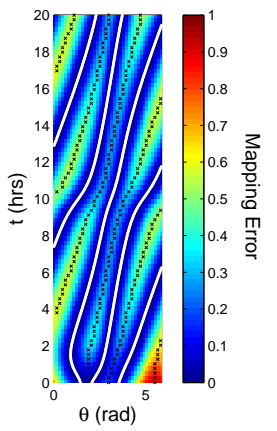

(c)
Fig. 5. Simulation of the sampling algorithm. The trajectories in (a) the transformed coordinates and (b) the original coordinates; (c) the corresponding error map.

coordinate transformation warps space and time near nonstationary locations, which clusters measurements in areas where the decorrelation scales are smaller. We regulate the inter-vehicle spacing using a phase-oscillator control strategy. We show that this control strategy corresponds to prescribing a steady-state sampling speed, which allows us to design vehicle trajectories using the same method as that used for stationary fields. We also provide the locations of local maximum error of the field. In ongoing work, the coverage strategy is being expanded to two spatial dimensions. We are also investigating the benefit of this algorithm as compared to other mapping error reduction strategies (e.g., Voronoi based approaches) to determine when it is a beneficial strategy.

\section{REFERENCES}

Bretherton, F.P., Davis, R.E., and Faundry, C.B. (1976). A technique for objective analysis and design of oceanographic experiments applied to MODE-73. Deep-Sea Res., 23(7), 559-582.

Cortez, J., Martinez, S., and Bullo, F. (2004). Coverage control for mobile sensing networks. IEEE Trans. on Robotics and Automation, 20(2), 243-255.

Davis, R.E., Leonard, N.E., and Fratantoni, D.M. (2009). Routing strategies for underwater gliders. Deep-Sea Research II, 56, 173-187.

Demetriou, M.A. (2010). Guidance of mobile actuatorplus-sensor networks for improved control and estimation of distributed parameter systems. IEEE Trans. on Automatic Control, 55(7), 1570-1584.

Eliassen, A. and Sawuer, J.S. (1954). Upper air network requirements for numerical weather prediction. Tech. Note. World Meteorological Org., 29.

Gandin, L.S. (1963). Gidrometerologicheskoe Izdatelstvo - Objective Analysis of Meteorological Fields. Israel Program for Scientific Translations.

Graham, R. and Cortes, J. (2009). Asymptotic optimality of multicenter Voronoi configurations for random field estimation. IEEE Trans. on Automatic Control, 54, $153-158$

Higdon, D., Swall, J., and Kern, J. (1999). Non-stationary spatial modeling. In Bayesian Statistics 6, 761-768. Oxford UP.

Inoue, J., Kawashima, M., Fujiyoshi, Y., and Wakatsuchi, M. (2005). Aircraft observations of air-mass modifi- cation over the Sea of Okhotsk during sea-ice growth. Boundary-Layer Meteorology, 117, 111-129.

Laventall, K. and Cortes, J. (2009). Coverage control my multi-robot networks with limited-range anisotropic sensory. Int. Journal of Control, 82, 1113-1121.

Leonard, N.E. and Olshevskyi, A. (2011). Nonuniform coverage control on the line. In Proc. of IEEE Conf. of Decision and Control, 3163-3168. Orlando, Florida.

Leonard, N.E., Paley, D.A., Davis, R.E., Fratantoni, D.M., Lekien, F., and Zhang, F. (2010). Coordinated control of an underwater glider fleet: An adaptive ocean sampling field experiment in Monterey Bay. J. Field Robotics, 27, 718-740.

Leonard, N.E., Paley, D.A., Sepulchre, R., Frantantoni, D.M., and Davis, R.E. (2007). Collective motion, sensor networks and ocean sampling. Proc. of the IEEE, 95(1), 48-74.

Liebelt, P.R. (1976). An Introduction to Optimal Estimation. Addison-Wesley.

Martinez, S., Cortes, J., and Bullo, F. (2007). Motion coordination with distributed information. IEEE Control Systems Magazine, 27, 75-88.

Paley, D.A. (2007). Cooperative Control of Collective Motion for Ocean Sampling with Autonomous Vehicles. Ph.D. thesis, Princeton University.

Paley, D.A. and Peterson, C. (2009). Stabilization of collective motion in a time-invariant flowfield. J. of Guidance, Control and Dynamics, 32(3), 771-779.

Rudnick, D.L. and Davis, R.E. (1988). Mass and heat budgets on the northern California continental shelf. $J$. Geophysical Research, 93(C11), 14013-14024.

Sampson, P.D. and Guttorp, P. (1992). Nonparametric estimation of nonstationary spatial covariance structure. J. of the American Statistical Association, 87, 108-1195.

Schmidt, A.M. and O'Hagan, A. (2003). Bayesian inference for non-stationary spatial covariance structure via spatial deformations. Journal of the Royal Statistical Society: Series B, 65, 743-758.

Sepulchre, R., Paley, D.A., and Leonard, N.E. (2007). Stabilization of planar collective motion: All-to-all communication. IEEE Trans. on Automatic Control, 52, 811-824.

Song, Z., Chen, Y., Liang, J., and Ucinski, D. (2005). Optimal mobile sensor motion planning under nonholonomic constraints for parameter estimation of distributed systems. In Proc. of IEEE Int. Conf. on Intelligent Robots and Systems, 3163-3168. Edmonton, Alberta, Canada.

Sydney, N. and Paley, D.A. (2011). Multi-vehicle control and optimization for spatiotemporal sampling. Proc. IEEE Conf. on Decision and Control, 5607-5612.

Torres, M., Campos, S., Pinto, E., Rajamani, S., Sayre, R., and Colepicolo, P. (2008). Biochemical biomarkers in algae and marine pollution: A review. Ecotoxicology and Environmental Safety, 71, 1-15.

Ucinski, D. and Demetriou, M. (2004). An approach to the optimal scanning measurement problem using optimum experimental design. In Proc. of the American Control Conf., volume 2, 1616-1621.

Zhang, F. and Leonard, N.E. (2010). Cooperative filters and control for cooperative exploration. IEEE Trans. on Automatic Control, 55, 650-663. 